\title{
Construction of a High-Quality Yeast One/Two-Hybrid cDNA Library from Cassava (Manihot Esculenta Crantz)
}

\author{
Jiao LIU ${ }^{1, a,{ }^{*} \text {, Shao-ping FU }}{ }^{1, a}$, Yu-qiang GUO ${ }^{1,2}$, Shuo WANG ${ }^{1,2}$, \\ Rui-jun DUAN ${ }^{1}$, Rui-mei LI ${ }^{1}$, Yuan YAO ${ }^{1}$ and Jian-chun GUO ${ }^{1}$ \\ ${ }^{1}$ Institute of Tropical Biosciences and Biotechnology, Chinese Academy of Tropical \\ Agricultural Sciences / Key Laboratory of Biology and Genetic Resources of Tropical \\ Crops, Ministry of Agriculture, Haikou 571101, Hainan, P.R. China \\ ${ }^{2}$ College of Agriculture, Hainan University, Haikou 570228, Hainan, P.R. China \\ a Jiao Liu and Shao-Ping Fu contributed equally to this work \\ *liujiao@itbb.org.cn \\ ${ }^{*}$ Corresponding author
}

Keywords: cDNA Library, Yeast One-Hybrid, Cassava.

\begin{abstract}
Cassava is an important food and potential biofuel crop with starch-rich edible tuberous roots. It is tolerant to multiple abiotic stresses and can produce an acceptable yield under the adverse climatic and nutrient-poor soil. However, the molecular mechanisms of starch accumulation and the abiotic stress response involved in cassava are less known. In the present study, total RNA was isolated from the mixed leaves, stems, flowers, fruits and storage roots of cassava, and a good quality cDNA library was constructed. The titers of the resultant cDNA library were $\sim 1.15 \times 10^{7} \mathrm{pfu} / \mathrm{ml}$ and an average insert size of $\sim 1.5 \mathrm{~kb}$. Therefore, the cDNA library constructed in this study was highly efficient. It can be used in the yeast one/two hybrid systems to investigate both known and new interactions between DNA sequence of interest and transcription factors involved in the metabolic pathways and signaling transduction pathways of cassava.
\end{abstract}

\section{Introduction}

Cassava (Manihot esculenta Crantz) is the euphorbiaceae dicotyledonous plants, which originated in South America amazon river basin. Cassava is a typical C3 plant, but has the characteristics of $\mathrm{C} 4$ plants photosynthesis [1]. Cassava root can accumulate a large amount of starch, considered as an important food and potential biofuel crop for production of ethanol and bioenergy [2]. Moreover, cassava can be tolerant to multiple abiotic stresses, such as drought, high temperature, low nitrogen and phosphorus nutrient-poor soil, but sensitive to cold [3]. However, the molecular mechanisms of starch accumulation and the abiotic stress response involved in cassava are currently less known.

Protein-protein interaction networks and the interaction of proteins with DNA controls almost all aspects of cellular function, such as recombination, replication, DNA repair, transcriptional regulation and chromosome maintenance. Yeast twohybrid assay $(\mathrm{Y} 2 \mathrm{H})$ is a powerful and high-throughput protein-protein screen system to identify or mine the partners of proteins in the regulatory complexes [4]. While yeast one-hybrid assay $(\mathrm{Y} 1 \mathrm{H})$, a modification of the yeast-two hybrid assay, is a sensitive technique for identifying and analyzing proteins that bind to a specific DNA fragment of the interest, and used to detect both known and new interactions between DNA sequence of interest and transcription factors [5]. 
In this study, we report the construction of a high quality of cDNA library from cassava mixed leaves, stems, flowers, fruits and storage roots. The cDNA library can be used in the yeast one/two -hybrid systems to investigate the proteins interaction or the interactions between DNA sequence of interest and transcription factors, which involved in the metabolic pathways and abiotic stress response related signaling transduction pathways of cassava.

\section{Materials and Methods}

\section{Plant Materials}

Manihot esculenta SC8 is a widely planted cassava cultivar in China, which were grown in the Chinese Academy of Tropical Agricultural Sciences (Haikou, China). Young leaves, stems, flowers, fruits and storage roots were harvested from Manihot esculenta SC8 in the field.

\section{Total RNA and mRNA Isolation}

Total RNA was extracted using Trizol (Invitrogen) from mixed leaves, stems, flowers, fruits and storage roots. Total RNA was treated with RNase-free DNase (Fermentas) to remove genomic DNA contamination. mRNA was enriched from total RNA by using Oligotex mRNA Kits (Qiagen) as per manufacturer's instruction.

\section{cDNA Synthesis and Purification}

The poly A+ mRNA enriched from total RNA were used to synthesize the first-strand cDNA with CDS III Primer, SMART MMLV RT and SMART III oligo. Then the resultant first-strand single-strand cDNAs were exponentially amplified by long distance PCR (LD-PCR) using the Advantage 2 PCR Kit (Clontech) to synthesize double-strand cDNA (ds cDNA) following the manufacturer's instruction. The PCR reactions along with a $1 \mathrm{~kb}$ DNA ladder were analyzed on a $1.2 \%$ agarose gel. The high quality ds cDNAs were purified with CHROMA SPIN+ TE-400 column containing the equilibration buffers were centrifuged at $700 \mathrm{~g}$ for $5 \mathrm{~min}$ to keep the matrix semi-dry, applied a ds cDNA sample to each column at the center of the flat surface of the gel matrix and centrifuged at $700 \mathrm{~g}$ for $5 \mathrm{~min}$. The purified ds cDNAs collected in the collection tubes were precipitated with 1/10th vol $3 \mathrm{M}$ sodium acetate (pH 5.3) and 2.5 vol of ice-cold ethanol (95-100\%). Placed in $-20^{\circ} \mathrm{C}$ freezer for $1 \mathrm{hr}$ then centrifuged at $14,000 \mathrm{rpm}$ for $20 \mathrm{~min}$ at room temperature and removed remaining supernatant. Air dry the cDNA pellet for $10 \mathrm{~min}$ and resuspended the cDNA in $20 \mu \mathrm{l}$ deionized water. The purified ds cDNAs were ready for library construction.

\section{cDNA Library Construction}

The purified ds DNAs infused with the linearized pGADT7-Rec AD cloning vector (Clontech), the recombinants were co-transformed into E.coli competent cells. After 225-250 rpm cultured $1 \mathrm{hr}$ at $37^{\circ} \mathrm{C}, 10 \mu \mathrm{l}$ transformed cultures diluted to 100 -fold were plated on LB (with $50 \mu \mathrm{g} / \mathrm{ml}$ ampicillin) plates. The number of clones was counted to calculate the library titer using the formula: cfu/ml=number of plaques/10 $\mu 1 \times$ dilution factor $\times 10^{3} \mu 1 / \mathrm{ml}$ ( $\mu$ l of diluted phage plated). The size of the insert fragment was confirmed by the PCR using random selection of 20-30 clones. The cDNA library bacteria cultures were plated on the $35 \mathrm{~cm}$ LB plates containing 10000-20000 clones for 1 day, extracted cDNA library plasmids from the mixed clones. 


\section{Results and Discussion}

\section{Total RNA Isolation and LD-PCR}

The RNA quality is the critical factor to construct a high quality cDNA library [6]. Total RNA from cassava mixed leaves, stems, flowers, fruits and storage roots was extracted and treated with RNase-free DNase (Fermentas) to remove genomic DNA contamination. The A260/A280 ratio of isolated RNA was found to be 1.90, agarose gel electrophoresis of isolated RNA sample showed clear distinct band of $28 \mathrm{~S}$ and $18 \mathrm{~S}$, confirming good quality of isolated RNA (Figure 1a).

To get enough long clone and increase absolute numbers of primary clones, we used a high quality reverse transcriptase enzyme MMLV of SMART technology and high-quality LD-PCR to synthesize adequate ds cDNA from the isolated cassava RNA (Figure 1b). We also adopted Column Chromatography technology (CHROMA SPIN TE-400, Clontech) to purify the ds cDNA and perform cDNA size fractionation, the ds cDNAs with DNA molecules > 400-600 bp were collected (Figure 1c).

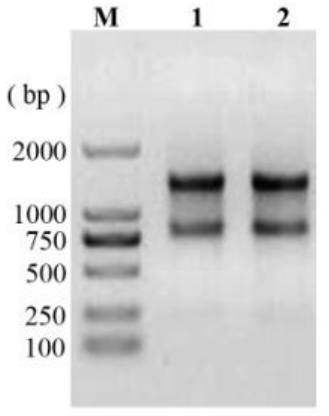

(a)

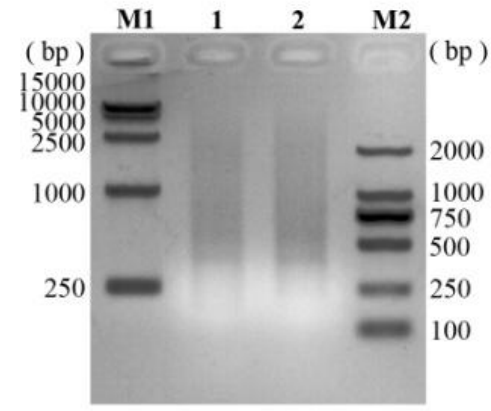

(b)

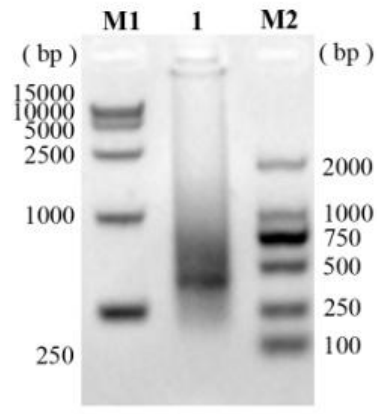

(c)

Figure 1. Isolation of cassava total RNA and synthesis of purified ds cDNA.

(a) Isolation of total RNA; (b) Synthesis of ds cDNA; (c) Purification of ds cDNA.

\section{Constructon and Quality Test of cDNA Library}

The purified ds DNAs infused with the linearized pGADT7-Rec AD cloning vector were co-transformed into E.coli competent cells and cultured on LB plates (with $50 \mu \mathrm{g} / \mathrm{ml}$ ampicillin). $10 \mu \mathrm{l}$ transformed cultures diluted to 100 -fold on LB plate obtained 230 primary clones, the total volume of transformed cultures as $5 \mathrm{ml}$, showing that the capacity of cDNA library was $230 / 10 \times 100 \times 1000 \times 5=1.15 \times 10^{7} \mathrm{cfu}$.

Meanwhile, we also checked the sizes of inserts in "prey" vectors (Figure 2). The average size of inserts in our cDNA library was about $1.5 \mathrm{~kb}$ and the positive rate was $100 \%$, which was comparable in insert size to the reported high quality cDNA library [6]. Therefore, the cDNA library was constructed successfully and expected to perform efficient $\mathrm{Y} 2 \mathrm{H}$ or $\mathrm{Y} 1 \mathrm{H}$ screens. 


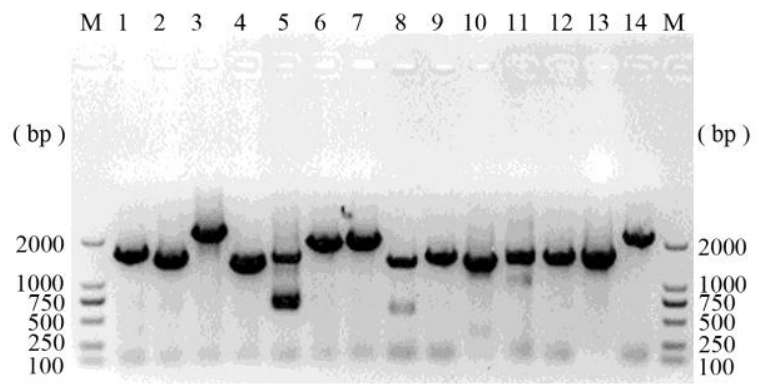

Figure. 2. A mplified parts of inserts of cDNA clones from the constructed cDNA library.

M: DNA size marker; Lanes 1-14: Insert cDNA clones in phage plaques.

\section{Acknowledgme nts}

This work was supported by the National Natural Science Foundation of China (31371706); Young Talent Cultivation Project of China Association for Science and Technology; the Earmarked Fund for Modern Agro-industry Technology Research System (CARS-12-hnwwq).

\section{References}

[1] Angelov MN, Sun J, Byrd GT, Black CC. Novel characteristics of cassava, Manihot esculenta Crantz, a reputed C3-C4 intermediate photosynthesis species. Photosynth Res, 38(1): 61-72 (1993).

[2] Zidenga T, Leyva-Guerrero E, Moon H, Siritunga D, Sayre R. Extending cassava root shelf life via reduction of reactive oxygen species production. Plant Physiol, 159: 1396-1407 (2012).

[3] Xu J, Duan X, Yang J, Beeching JR, Zhang P. Enhanced reactive oxygen species scavenging by overproduction of superoxide dismutase and catalase delays postharvest physiological deterioration of cassava storage roots. Plant Physiol, 161: 1517-1528 (2013).

[4] Miller J, Stagljar I. Using the Yeast Two-Hybrid System to Identify Interacting Proteins. Methods Mol Biol, 261: 247-262 (2004).

[5] Bipasha Dey, Sameer Thukral, Shruti Krishnan, Mainak Chakrobarty, Sahil Gupta, Chanchal Manghani, Vibha Rani. DNA-protein interactions: methods for detection and analysis. Mol Cell Biochem, 365:279-299 (2012).

[6] Cao SH, Siriwardana CL, Kumimoto RW, Holt BF. Construction of high quality Gateway (TM) entry libraries and their application to yeast two-hybrid for the monocot model plant Brachypodium distachyon. BMC Biotechnol, 11:53 (2011). 